\title{
Sistema de Vigilância de Violências e Acidentes/VIVA e a notificação da violência infanto-juvenil, no Sistema Único de Saúde/SUS de Feira de Santana-Bahia, Brasil
}

\author{
Surveillance System for Violence and Accidents (VIVA) and \\ notification of infant-juvenile violence in the Brazilian Unified \\ Health System (SUS) in Feira de Santana in the state of Bahia
}

Camila dos Santos Souza ${ }^{1}$

Maria Conceição Oliveira Costa ${ }^{1}$

Simone Gonçalves de Assis ${ }^{2}$

Jamilly de Oliveira Musse ${ }^{1}$

Carlito Nascimento Sobrinho ${ }^{1}$

Magali Teresópolis Reis Amaral ${ }^{1}$

\footnotetext{
${ }^{1}$ Programa de PósGraduação em Saúde Coletiva, Núcleo de Estudos e Pesquisas na Infância e Adolescência, Departamento de Saúde, Universidade Estadual de Feira de Santana. Av. Transnordestina S/N Campus Universitário - Módulo VI, Novo Horizonte. 44.031-460 Feira de Santana BA Brasil. camila_s.souza@hotmail.com ${ }^{2}$ Centro Latino-Americano de Estudos de Violência e Saúde Jorge Careli, ENSP, Fiocruz.
}

Abstract The scope of the study is to analyze data of children and adolescents who are the victims of different forms of violence, registered in the Surveillance System for Violence and Accidents (VIVA/MS) in Feira de Santana in the state of Bahia, Brazil. The total number of records, since the implementation of VIVA in the city (01/2009 to 01/2011) was used and the analyses sought associations between characteristics of violence and profiles of victims and perpetrators. The results showed that children and adolescents were molested using different types of violence, including the use of physical force, verbal threats and weapons and sundry cases of personal injury. Approximately 35\% were hospitalized and 15\% died. Physical violence was more common among males during adolescence in the home environment and perpetrated by a family member. Sexual violence occurred with greater frequency among females during childhood and $55.5 \%$ of the cases occurred in the home environment and more frequently perpetrated by a family member or acquaintances. The results highlight the importance of investments in policies and programs for harm prevention and reduction, seeking to broaden coverage in the care and the enhancement of the information system and surveys of these indicators.

Key words Violence, Children, Adolescents, Information systems, Epidemiological surveillance Violence, Children, Adolescents, Information systems, Epidemiological surveillance
Resumo O estudo tem como objetivo analisar os dados de crianças e adolescentes vitimas das distintas formas de violência, registrados no Sistema de Vigilância de Violências e Acidentes/VIVA/MS, de Feira de Santana, Bahia, Brasil. Utilizou-se o total de registros, desde a implantação do Sistema VIVA no município (01/2009 a 01/2011), cujas análises buscaram associações entre características das violências e perfis de vítimas e agressores. Os resultados mostraram que crianças e adolescentes foram molestados por diversas violências; com uso de força corporal, ameaça verbal e armas; ocorrência de lesões corporais diversas; aproximadamente 35\% foram hospitalizados e 15\% evoluíram a óbito; a violência física apresentou maior frequência no sexo masculino, nas faixas da adolescência, ocorrência em ambiente domiciliar e causado por familiar; a violência sexual ocorreu com maior proporção no sexo feminino, nas faixas da infância, 55,5\% das ocorrências foram em nível domiciliar, sendo mais frequentemente perpetrada por conhecidos e familiares. Os resultados sugerem a importância de investimentos em politicas e programas de prevenção e redução de danos, buscando ampliar a cobertura no atendimento e aprimoramento do Sistema de Informação e levantamento desses indicadores.

Palavras-chave Violência, Crianças, Adolescentes, Sistemas de informação, Vigilância Epidemiológica 


\section{Introdução}

Em nível mundial, a violência tem sido reconhecida como importante questão social e de saúde pública. Ao publicar o Relatório Mundial sobre Violência, a Organização Mundial da Saúde (OMS) coloca o problema como desafio universal e traz a discussão para a área da saúde que, tradicionalmente, lidava apenas com as consequências dos eventos ${ }^{1}$. Nessa perspectiva, estudiosos apontam para a importância da integração desse setor na Rede de Prevenção e Enfrentamento, considerando o impacto positivo da interdisciplinaridade e intersetorialidade, no desenvolvimento de estratégias direcionadas aos grupos populacionais mais vulneráveis ${ }^{2-4}$.

Os acidentes e as violências na infância e na adolescência envolvem peculiaridades marcantes em relação à faixa etária, ao local de ocorrência e às características ou circunstâncias em que se desenvolvem. Na medida em que aumentam os estudos sobre acidentes e violências, identificando os fatores e os processos da sua ocorrência, as circunstâncias e o ambiente social em que ocorre, essa realidade tende a mudar, ou pelo menos, a tornar-se mais visível e, desta forma, a evoluir no sentido de redução da impunidade e de proteção das vítimas ${ }^{5}$.

No Brasil, a articulação entre as diferentes áreas do conhecimento visa o cumprimento de princípios assegurados pelo Estatuto da Criança e do Adolescente (ECA), quanto às políticas e programas. Os profissionais representam agentes de proteção, com o encargo de notificar os casos suspeitos ou confirmados no Sistema de Vigilância de Acidentes e Violência/VIVA do Ministério da Saúde e encaminhar à Instância de referência para denúncias ao Sistema de Garantia de Direitos da Criança e Adolescente (SGDCA) - Conselhos Tutelares da respectiva localidade $e^{4,6,7}$.

Em nível nacional, o Sistema de Saúde realiza o monitoramento das violências, para fins de vigilância epidemiológica, através da Declaração de Óbito (DO) e Autorização de Internação em Hospitais (AIH), fornecidos, respectivamente, pelo Sistema de Informações sobre Mortalidade (SIM) e Sistema de Informações Hospitalares (SIH), do Sistema Único de Saúde (SUS), gerenciados pelo Ministério da Saúde (MS), os quais registram características dos casos violentos, com desfecho para óbito ou internação. Contudo, essas fontes não captam as lesões de menor gravidade, as quais são responsáveis por grande demanda nos serviços de emergência. Visando levantar indicadores de morbidade dos eventos que não resultam em internações ou óbitos, em 2006 o MS implantou o Sistema VIVA, em toda Rede de Serviços do SUS, na perspectiva de viabilizar e divulgar informações sobre casos de violência nos diversos segmentos populacionais e cursos da vida ${ }^{8}$.

O Sistema VIVA encontra-se organizado em dois componentes: a) contínuo, que integra o "Sistema de Informação de Agravos de Notificação", por meio da notificação compulsória realizada através dos serviços de saúde, em nível nacional; b) inquérito, realizado por meio de estudo transversal, em serviços de urgência e emergência selecionados, no período de trinta dias a cada dois anos?.

Na perspectiva da atuação no Sistema de Saúde e diante de situações de violência, o profissional tem responsabilidades nos aspectos jurídicos e da consciência moral. Embora o reconhecimento das vítimas e a atuação no enfrentamento da violência façam parte das atribuições desses profissionais, muitas vezes os mesmos ainda não se encontram familiarizados com os aspectos legais a serem adotados diante dos casos, sobretudo no tocante à notificação ${ }^{10}$.

A notificação dos casos de violência pelos profissionais de saúde transcende os limites do serviço, cujo objetivo é interromper a cadeia de eventos e consequências ${ }^{11}$. $\mathrm{O}$ caso identificado exige a intervenção interdisciplinar e parcerias institucionais, cujas ações são imprescindíveis para a resolutividade dos casos. A partir da notificação, desencadeia-se a investigação da família, dos ciclos de convivência social, procedimento que primordialmente constitui uma importante estratégia de cuidado e proteção às vítimas, além do que representa um poderoso instrumento de política pública, uma vez que ajuda a dimensionar a questão e determinar a necessidade de investimentos em núcleos de vigilância e assistência ${ }^{10}$.

Em nível nacional, estudo multicêntrico realizado com dados do Sistema VIVA (2010), com objetivo de avaliar a distribuição das notificações de violência doméstica, sexual e outras, perpetradas em menores de 10 anos, segundo as unidades da federação (UF), observou que o Estado da Bahia tem uma das mais baixas taxas de notificação e são poucos os municípios baianos (apenas onze), cujos Serviços de Saúde têm o Sistema VIVA implantado, no período investigado ${ }^{12}$.

Cabe enfatizar que esse processo tem impacto fundamental nas medidas de prevenção e enfrentamento, assim como no monitoramento de dados e levantamento de indicadores que permitem quantificar e caracterizar os múltiplos as- 
pectos da violência, nos diversos contextos e grupos sociais. O planejamento e o desenvolvimento de ações com base nesses índices permitem aos serviços acompanharem os casos, bem como reivindicar mudanças políticas, envolvendo cumprimento de direitos, responsabilização, integração da rede de serviços, prevenção e promoção da saúde ${ }^{13}$.

Não obstante os avanços das pesquisas sobre caracterização das ações em vigilância vale salientar as limitações de estudos sobre as notificações, em nível nacional e municipal. Estudos restritos a dados de serviços específicos, mais comuns, não permitem dimensionar o problema e avaliar o impacto da notificação e dos encaminhamentos decorrentes do processo, nos diferentes contextos e regiões. A potencialidade do presente estudo sustenta-se no levantamento e divulgação de indicadores municipais, em região considerada de alta vulnerabilidade aos agravos sociais, como a violência, viabilizando a comparabilidade de dados, tendo em vista a variedade de contextos socioeconômicos e políticos das cidades brasileiras. Visa ainda subsidiar a compreensão dos diferentes aspectos desse fenômeno e a análise do processo de notificação; assim como contribuir com o poder público, na implementação de ações estratégias de prevenção e intervenção.

No que diz respeito à participação de Feira de Santana no levantamento de indicadores, vale ressaltar que o município sede desta pesquisa integra o grupo de cidades consideradas de risco para violação, tráfico e exploração de crianças e adolescentes, pelas características econômicas e geográficas: polo econômico e industrial do estado, portal da região do semiárido do estado da Bahia e situada em um dos importantes trevos rodoviários do país, ligação entre as regiões norte, nordeste e sudeste do país (através de rodovias federais e estaduais), com alto fluxo migratório das regiões mais pobres, em direção às maiores cidades e a capital Salvador, importante polo turístico do país. O município integra o Programa Nacional de Enfrentamento da Violência infanto-juvenil, desde 2003, momento no qual assinou o pacto de participação e implantação da Rede de Atendimento e Garantia de Direitos, integrando Instâncias e Universidade, entretanto, o Sistema VIVA começou a ser implantado no Sistema de Municipal de Saúde, a partir de 2009, sob a responsabilidade do Setor de Vigilância Epidemiológica.

Este artigo tem como objetivo analisar os dados de vitimização de crianças e adolescentes, pelas distintas formas de violência, registrados na Rede de Atendimento do SUS, do Sistema de Vigilância de Violências e Acidentes/VIVA do município de Feira de Santana (BA), no período 01/2009 a 01/2011.

\section{Método}

Estudo descritivo realizado com dados secundários coletados das fichas de notificação do Sistema de Vigilância de Violências e Acidentes/VIVA, referente aos casos de crianças e adolescentes vítimas de diferentes formas de violência, atendidos nas Instâncias do Sistema Único de Saúde/ SUS (Unidades Básicas, Programa de Saúde da Família, Policlínicas e Hospitais) de Feira de Santana, desde a implantação, no período 01-01-2009 a 01-01-2011.

$\mathrm{O}$ acesso aos dados deu-se no Serviço de Vigilância Epidemiológica/Secretaria Municipal de Saúde de Feira de Santana (Núcleo de Violências e Acidentes). Os dados foram classificados, processados e analisados como constam na ficha de notificação do Sistema VIVA (notificação individual, tipologia da violência, lesão, dados do agressor, evolução e encaminhamentos).

Para maior compreensão e limpeza dos dados contidos na ficha, foi criado um novo questionário, reorientando os dados pertinentes para análise, principalmente as informações relativas à tipologia da violência, violência sexual e lesões, possibilitando uma nova digitação com critérios rigorosos. Optou-se pela pesquisa de campo em ficha específica e não pela coleta de dados diretamente do SINAN-NET, em função das limitações do referido sistema e prejuízos relativos à subnotificação.

As variáveis foram organizadas segundo $d a$ dos das vítimas (sexo, faixa etária, escolaridade); dados do agressor (números de envolvidos; sexo; uso de álcool e vínculo com a vítima); característica da violência (tipologia, meio de agressão, natureza da lesão, parte do corpo comprometida, local de ocorrência, recorrência e consequências); evolução e encaminhamentos para setor saúde e outros setores; dados da violência sexual (penetração sexual; procedimentos realizados). Os percentuais apresentados a seguir têm como base o total de respostas na questão, e não o total de casos investigados.

Os dados foram processados através do programa SPSS, versão 9.0. Foram realizadas análises bivariadas entre os diferentes tipos de violência, segundo variáveis das vítimas, agressores, local de ocorrência e recorrências. 
Essa pesquisa foi aprovada pelo Comitê de Ética em Pesquisa da Universidade Estadual de Feira de Santana/UEFS em atendimento aos princípios éticos da Resolução 196/96 ${ }^{14}$ do Conselho Nacional de Saúde.

\section{Resultados}

No período de 01/01/2009 a 01/01/2011, nas Instâncias que integram o Sistema Único de Saúde/ SUS do município, foram registrados 432 casos de violência entre crianças e adolescentes. As características das vítimas e agressores (Tabela 1) envolvidos nos diferentes tipos de violência mostraram proporções semelhantes de vitimização, no sexo masculino $(50,2 \%)$ e feminino $(49,8 \%)$; e faixas etárias da infância $(48,4 \%)$ e adolescência $(51,6 \%)$; Dentre os casos notificados com informação so-

Tabela 1. Características sociodemográficas das vítimas de violência e respectivos agressores, segundo o Sistema de Notificação de Violência e Acidentes।VIVA. Secretaria de Saúde de Feira de Santana 2009-2011.

\begin{tabular}{|c|c|c|}
\hline Características & $\mathbf{N}$ & $\%$ \\
\hline \multicolumn{3}{|l|}{ Vítimas } \\
\hline \multicolumn{3}{|l|}{ Sexo $(N=432)$} \\
\hline Masculino & 217 & 50,2 \\
\hline Feminino & 215 & 49,8 \\
\hline \multicolumn{3}{|l|}{ Idade (anos) $(\mathrm{N}=432)$} \\
\hline $0-11$ & 209 & 48,4 \\
\hline $12-18$ & 223 & 51,6 \\
\hline \multicolumn{3}{|l|}{ Agressor } \\
\hline \multicolumn{3}{|c|}{ Número de envolvidos $(\mathrm{N}=379)$} \\
\hline $\mathrm{Um}$ & 267 & 70,4 \\
\hline Dois ou mais & 112 & 29,6 \\
\hline \multicolumn{3}{|l|}{ Sexo $(N=379)$} \\
\hline Masculino & 269 & 71,0 \\
\hline Feminino & 69 & 18,2 \\
\hline Ambos os sexos & 41 & 10,8 \\
\hline \multicolumn{3}{|l|}{ Uso de álcool $(\mathrm{N}=223)$} \\
\hline $\operatorname{Sim}$ & 160 & 71,7 \\
\hline Não & 63 & 28,3 \\
\hline \multicolumn{3}{|c|}{ Vínculo com a vítima ${ }^{*}(\mathrm{~N}=377)$} \\
\hline Pais & 125 & 33,1 \\
\hline Outros familiares ${ }^{\mathrm{a}}$ & 68 & 18,0 \\
\hline Conhecido $^{\mathrm{b}}$ & 124 & 33,0 \\
\hline Desconhecido & 60 & 15,9 \\
\hline
\end{tabular}

Fonte: Sistema de Vigilância de Violências e Acidentes (VIVA), Secretaria de Saúde, Feira de Santana (BA). "As questões permitiam múltiplas escolhas; ${ }^{\mathrm{a} O u t r o s}$ familiares (padrasto, madrasta, irmãos, filhos, cônjuge parentes); ${ }^{\mathrm{b}}$ Conhecido (namorado, ex-namorado, amigo, patrão). bre o agressor totalizam-se 379 indivíduos: a maioria, perpetrador único (70,4\%); do sexo masculino $(71,0 \%) ; 33,1 \%$ foram os pais; $33,0 \%$ conhecidos da vítima e 18,0\% outros familiares.

As análises entre os tipos de violência, características das vítimas, seu ambiente e respectivos agressores (Tabelas 2 e 3 ) mostraram perfis distintos entre as diversas manifestações da violência: para a violência física, foram verificadas maiores proporções no sexo masculino (55,6\%), nas faixas da adolescência $(55,6 \%)$, ocorridas em ambiente domiciliar (59,6\%), sem recorrências $(55,8 \%)$, causada por algum familiar $(57,4 \%)$ do sexo masculino (78,6\%); a violência sexual mostrou maiores proporções de vitimização no sexo feminino $(79,8 \%)$, nas faixas da infância $(53,7 \%)$, ocorrência domiciliar (55,5\%), com recorrências $(60,8 \%)$ e perpetrada por um conhecido $(62,8 \%)$ e familiar (37,2\%), do sexo masculino (95,5\%); a negligência ocorreu com maior frequência no sexo masculino (55,8\%), na infância $(84,4 \%)$, em ambiente domiciliar $(79,5 \%)$, com recorrência $(53,8 \%)$, perpetrada por familiar $(69,4 \%)$, do sexo masculino (67,2\%); a violência psicológica foi mais identificada no sexo feminino $(57,5 \%)$, na infância $(53,4 \%)$, ocorrência domiciliar $(75,1 \%)$, perpetrada por algum conhecido $(62,8 \%)$, do sexo masculino $(78,6 \%)$.

$\mathrm{Na}$ análise da tipologia, a violência física foi a mais frequente $(41,8 \%)$; os meios de agressão mais utilizados foram força corporal $(40,0 \%)$ e ameaça verbal $(32,8 \%)$, cabendo ressaltar $(14,0 \%)$ de utilização de arma de fogo. As lesões mais identificadas foram corte/laceração/perfuração $(45,7 \%)$; com maior proporção na região de face/crânio e pescoço $(31,5 \%)$ (Tabela 4$)$.

Ainda em relação ao perfil das ocorrências (Tabela 4), verificaram-se maiores proporções no domicílio $(62,4 \%)$ e ambiente social da vítima $(33,2 \%)$. Dentre o total de consequências relatadas nas notificações, destaca-se o estresse póstraumático $(83,8 \%)$, cabendo destacar $4,2 \%$ da ocorrência de gravidez e 9,0\% de DST. As recorrências ocorreram em mais de $50 \%$ dos casos, o encaminhamento hospitalar em $35,3 \%$ e em 15,3\% houve evolução fatal (óbito). Os setores mais requisitados para encaminhamentos foram Instâncias de Proteção e Atendimento (40,0\%), Centros de Referência $(25,8 \%)$ e Delegacias $(18,9 \%)$ (Tabela 4).

Quanto aos registros dos 118 casos de violência sexual (Tabela 5), é válido salientar que a ficha de notificação apresenta dois campos de notificação, onde 96 casos foram com penetração, sendo a maioria penetração genital $(89,6 \%)$. 
Tabela 2. Tipo de violência sofrida por crianças e adolescentes segundo características de vítimas e ocorrência. Sistema de Notificação de Violência e Acidentes (VIVA), Secretaria de Saúde. Feira de Santana 2009-2011.

\begin{tabular}{|c|c|c|c|c|c|c|c|c|}
\hline \multirow{2}{*}{ Características } & \multicolumn{2}{|c|}{ Física } & \multicolumn{2}{|c|}{ Sexual } & \multicolumn{2}{|c|}{ Negligência } & \multicolumn{2}{|c|}{ Psicológica } \\
\hline & $\mathbf{N}$ & $\%$ & $\mathbf{N}$ & $\%$ & $\mathbf{N}$ & $\%$ & $\mathbf{N}$ & $\%$ \\
\hline \multicolumn{9}{|l|}{ Vítimas } \\
\hline \multicolumn{9}{|l|}{ Sexo } \\
\hline Masculino & 158 & 55,6 & 25 & 20,7 & 43 & 55,8 & 82 & 42,5 \\
\hline Feminino & 126 & 44,4 & 96 & 79,3 & 34 & 44,2 & 111 & 57,5 \\
\hline \multicolumn{9}{|l|}{ Faixa etária (anos) } \\
\hline $0-11$ & 126 & 44,4 & 65 & 53,7 & 65 & 84,4 & 103 & 53,4 \\
\hline $12-18$ & 158 & 55,6 & 56 & 46,3 & 12 & 15,6 & 90 & 46,6 \\
\hline \multicolumn{9}{|l|}{ Ocorrência } \\
\hline \multicolumn{9}{|l|}{ Local } \\
\hline Residência & 149 & 59,6 & 61 & 55,5 & 58 & 79,5 & 139 & 75,1 \\
\hline Ambiente Social & 101 & 40,4 & 49 & 44,5 & 15 & 20,5 & 46 & 24,9 \\
\hline \multicolumn{9}{|l|}{ Recorrência } \\
\hline Sim & 99 & 44,2 & 62 & 60,8 & 35 & 53,8 & 121 & 70,3 \\
\hline Não & 125 & 55,8 & 40 & 39,2 & 30 & 46,2 & 51 & 29,7 \\
\hline
\end{tabular}

Fonte: Sistema de Vigilância de Violências e Acidentes (VIVA), Secretaria de Saúde, Feira de Santana (BA).

Tabela 3. Tipo de violência sofrida por crianças e adolescentes segundo características dos agressores. Sistema de Notificação de Violência e Acidentes (VIVA), Secretaria de Saúde. Feira de Santana 2009-2011.

\begin{tabular}{|c|c|c|c|c|c|c|c|c|}
\hline \multirow{3}{*}{$\begin{array}{l}\text { Características } \\
\text { do agressor }\end{array}$} & \multicolumn{8}{|c|}{ Tipo de violência } \\
\hline & \multicolumn{2}{|c|}{ Física } & \multicolumn{2}{|c|}{ Sexual } & \multicolumn{2}{|c|}{ Negligência } & \multicolumn{2}{|c|}{ Psicológica } \\
\hline & $\mathbf{N}$ & $\%$ & $\mathbf{N}$ & $\%$ & $\mathbf{N}$ & $\%$ & $\mathbf{N}$ & $\%$ \\
\hline \multicolumn{9}{|l|}{ Sexo } \\
\hline Masculino & 173 & 78,6 & 105 & 95,5 & 41 & 67,2 & 121 & 78,6 \\
\hline Feminino & 47 & 21,4 & 5 & 4,5 & 20 & 32,8 & 33 & 21,4 \\
\hline \multicolumn{9}{|l|}{ Uso de álcool } \\
\hline Sim & 45 & 32,1 & 13 & 22,0 & 13 & 27,1 & 36 & 34,0 \\
\hline Não & 95 & 67,9 & 46 & 78,0 & 35 & 72,9 & 70 & 66,0 \\
\hline \multicolumn{9}{|l|}{ Vínculo com vítima } \\
\hline Familiares $^{*}$ & 97 & 57,4 & 35 & 37,2 & 43 & 69,4 & 35 & 37,2 \\
\hline Conhecidos & 72 & 42,6 & 59 & 62,8 & 19 & 30,6 & 59 & 62,8 \\
\hline
\end{tabular}

Fonte: Sistema de Vigilância de Violências e Acidentes (VIVA), Secretaria de Saúde, Feira de Santana (BA). * Familiares: inclui pais e parentes.

Os procedimentos relatados em 67 casos foram as profilaxias para Hepatite B, DST, HIV, além das medidas contraceptivas $(55,2 \%)$ e exames laboratoriais $(44,7 \%)$.

\section{Discussão}

A vitimização de crianças e adolescentes tem ocupado destaque nas agendas de organizações in- ternacionais e políticas nacionais, compondo um trágico painel a ser enfrentado pela sociedade.

A promulgação do Estatuto da Criança e Adolescente/ECA, a implantação dos Conselhos Tutelares, a organização dos sistemas locais de saúde e a criação dos serviços de prevenção de violência têm contribuído para o aumento da notificação e registro da violência infanto-juvenil, assim como estimulado o controle social, com vistas à interrupção do ciclo de vitimização - agressão ${ }^{15-17}$. 
Tabela 4. Características dos casos de violência em crianças e adolescentes, encaminhamentos e evolução, segundo registros do Sistema de Notificação de Violência e Acidentes।VIVA. Secretaria de Saúde de Feira de Santana 2009-2011.

\begin{tabular}{|c|c|c|}
\hline Características das violências & $\mathbf{N}$ & $\%$ \\
\hline \multicolumn{3}{|l|}{ Tipo de violência* $(\mathrm{N}=684)$} \\
\hline Física & 285 & 41,7 \\
\hline Sexual & 121 & 17,7 \\
\hline Negligência/Abandono & 77 & 11,3 \\
\hline Psicológica & 193 & 28,2 \\
\hline Outras $^{\mathrm{a}}$ & 8 & 1,2 \\
\hline \multicolumn{3}{|l|}{ Meio de agressão* $(N=430)$} \\
\hline Força corporal & 172 & 40,0 \\
\hline Objetos $^{\mathrm{b}}$ & 44 & 10,2 \\
\hline Envenenamento & 13 & 3,0 \\
\hline Arma de fogo & 60 & 14,0 \\
\hline Ameaça verbal & 141 & 32,8 \\
\hline \multicolumn{3}{|l|}{ Tipo de lesão* $(\mathrm{N}=221)$} \\
\hline Corte/perfuração/laceração & 101 & 45,7 \\
\hline Entorse/luxação/contusão & 50 & 22,6 \\
\hline Traumatismo & 27 & 12,2 \\
\hline Intoxicação & 17 & 7,7 \\
\hline Outros $^{c}$ & 26 & 11,8 \\
\hline \multicolumn{3}{|l|}{ Parte do corpo atingida ${ }^{*}(\mathrm{~N}=257)$} \\
\hline Crânio/face/pescoço & 81 & 31,5 \\
\hline Tronco & 43 & 16,7 \\
\hline Membros Superiores e Inferiores & 36 & 14,0 \\
\hline Órgãos genitais & 63 & 24,5 \\
\hline Múltiplas regiões & 34 & 13,2 \\
\hline \multicolumn{3}{|l|}{ Local da ocorrência $(\mathrm{N}=388)$} \\
\hline Residência & 242 & 62,4 \\
\hline Instituição de ensino e amparo & 17 & 4,4 \\
\hline Ambiente Social & 129 & 33,2 \\
\hline \multicolumn{3}{|l|}{ Consequência da violência* $(\mathrm{N}=141)$} \\
\hline Aborto & 1 & 0,7 \\
\hline Gravidez & 6 & 4,2 \\
\hline DST & 13 & 9,3 \\
\hline Tentativa de suicídio & 3 & 2,1 \\
\hline Estresse pós-traumático & 119 & 83,8 \\
\hline \multicolumn{3}{|l|}{ Recorrência $(\mathrm{N}=347)$} \\
\hline Sim & 186 & 53,6 \\
\hline Não & 161 & 46,4 \\
\hline \multicolumn{3}{|l|}{ Características dos encaminhamentos e evolução } \\
\hline \multicolumn{3}{|l|}{ Encaminhamento no setor saúde $(\mathrm{N}=266)^{3}$} \\
\hline Ambulatorial & 172 & 64,7 \\
\hline Internação hospitalar & 94 & 35,3 \\
\hline \multicolumn{3}{|l|}{ Encaminhamento da vítima para outros setores ${ }^{*}(\mathrm{~N}=745)$} \\
\hline Instituição de Proteção e Atendimento à criança e o adolescente & 298 & 40,0 \\
\hline Delegacias e Judiciário & 141 & 18,9 \\
\hline Centros de Referências & 192 & 25,8 \\
\hline Instituto Médico Legal & 38 & 5,1 \\
\hline Outros $^{\mathrm{d}}$ & 76 & 10,2 \\
\hline \multicolumn{3}{|l|}{ Evolução dos casos $(\mathrm{N}=307)$} \\
\hline Alta & 238 & 77,5 \\
\hline Evasão/Fuga & 22 & 7,2 \\
\hline Óbito & 47 & 15,3 \\
\hline
\end{tabular}

Fonte: Sistema de Vigilância de Violências e Acidentes (VIVA), Secretaria de Saúde, Feira de Santana (BA). *As questões permitiam múltiplas escolhas; ${ }^{\mathrm{a}}$ Outras (intervenção policial e trabalho infantil); ${ }^{\mathrm{b}}$ Objetos (perfuro-cortante, contundente e quente); ${ }^{\mathrm{c}}$ Outros (queimadura e amputação); ${ }^{\mathrm{d}}$ (Hospital, programa de DST/HIV/AIDS). 
Tabela 5. Características dos casos de violência sexual de crianças e adolescentes, segundo o Sistema de Notificação de Violência e Acidentes/VIVA. Secretaria de Saúde de Feira de Santana 2009-2011.

\begin{tabular}{|c|c|c|}
\hline Violência sexual & $\mathbf{N}$ & $\%$ \\
\hline \multicolumn{3}{|l|}{$\operatorname{Tipo}^{*}(\mathrm{~N}=118)$} \\
\hline Abuso sexual & 104 & 88,1 \\
\hline Exploração sexual $^{\mathrm{a}}$ & 14 & 11,9 \\
\hline \multicolumn{3}{|l|}{ Penetração* $^{*}(\mathrm{~N}=96)$} \\
\hline Oral & 10 & 10,4 \\
\hline Genital & 86 & 89,6 \\
\hline \multicolumn{3}{|c|}{ Procedimento realizado ${ }^{*}(\mathrm{~N}=67)$} \\
\hline Profilaxias ${ }^{\mathrm{b}}$ & 37 & 55,2 \\
\hline Exames laboratoriais & 30 & 44,8 \\
\hline
\end{tabular}

Fonte: Sistema de Vigilância de Violências e Acidentes (VIVA), Secretaria de Saúde, Feira de Santana (BA). "As questões permitiam múltiplas escolhas; ${ }^{\text {a}}$ Exploração sexual (envolvimento de comercio - trocas materiais); ${ }^{\mathrm{b}}$ Profilaxias (DST, HIV, Hepatite B e contracepção de emergência).

Os registros do Sistema VIVA, por ser este uma estratégia de vigilância de acidentes e violências em parceria com Estados e Municípios, têm sido utilizados no nível local para o melhor conhecimento da violência infligida contra crianças e adolescentes. Essa ferramenta tem facilitado articulações entre os sistemas de saúde e de garantia de direitos, responsáveis pela notificação (Conselhos Tutelares) e toda a rede de referência, integrando ações de promoção à saúde, prevenção e controle das violências ${ }^{18}$. O VIVA contribui para ampliar o conhecimento da violência-infanto-juvenil, na perspectiva do Setor Saúde, auxiliando no levantamento de indicadores, assim como subsidiando a ampliação das políticas, programas e práticas voltadas à prevenção, enfrentamento da violência e redução de danos. Neste sentido, contribui para a consolidação da Rede de atenção integral às vítimas de violência, cabendo enfatizar a importância da articulação do Sistema de Saúde com a Rede de Atendimento e Defesa dos Direitos, diante do desafio de identificar, notificar e encaminhar os casos, ampliando a resolutividade e garantindo proteção e defesa de direitos, especialmente nos grupos mais vulneráveis e menos favorecidos.

A qualidade da informação, ou seja, a completude no preenchimento dos campos da ficha, bem como a adesão de profissionais de saúde podem ser considerados pontos críticos ao Sistema de Vigilância associado à subnotificação dos dados hoje ainda existentes. O treinamento das equipes, com o objetivo de reforçar a importân- cia da notificação, também necessita ser incrementado visando à melhoria do Sistema ${ }^{15}$.

A análise dos dados do VIVA em Feira de Santana tem relevância singular pelas características marcantes quanto ao fenômeno violência, justificada por sua geografia local (entroncamento rodoviário), onde deságuam e confluem cenários propícios para a exploração sexual e a violência urbana, realidade presente em outras regiões brasileiras reiterando a importância de estudos com dados locais.

Em Feira de Santana, a análise da tipologia do evento, pelo Sistema VIVA, apontou maior frequência da violência física na faixa da adolescência, com vítima do sexo masculino e agressor na categoria de familiar, enquanto as maiores proporções de violência sexual e psicológica ocorreram no sexo feminino, na faixa da infância, perpetrada por familiar e conhecido da vítima. Esses achados assinalam o alto impacto da vitimização perpetrada pela família, assim como a interferência de aspectos extrafamiliares, considerando a alta frequência de agressor conhecido da vítima, podendo ser amigos, vizinhos e conhecidos, com acesso ao domicílio e convívio social. Esses resultados concordam com estudos que sugerem a importância do ambiente e do relacionamento interpessoal, na dinâmica e estilo de vida das famílias, em diferentes segmentos sociais e contextos ${ }^{11,15}$.

Em outros trabalhos publicados envolvendo dados do sistema VIVA com a população infanto-juvenil, observa-se que as agressões físicas foram o tipo mais comum de violência, sendo mais comum de 6 a 9 anos e as negligências mais freqüentes em crianças de 2 a 5 anos, com mais da metade dos casos com agressor familiar ${ }^{19}$. Redirecionando a análise na categoria juvenil de 10 a 19 anos, os autores revelam que dentre os participantes $6.434(89,9 \%)$ foram vítimas de acidentes e $730(10,2 \%)$ de violências ${ }^{19}$. O tipo de violência também mais comum foi a violência física, especificada pela agressão em 90,3\% dos casos, com agressor familiar predominantemente na faixa etária de 10 a $14 \operatorname{anos}^{5}$. Os dados nacionais correlacionados com os encontrados no presente estudo corroboram com perfil de morbimortalidade de nosso país, motivadas pelas causas externas.

No que concerne à violência física, estudiosos apontam que fatores culturais, ligados às questões de poder e de gênero propiciam à agressão física, castigos corporais, como forma de resolver conflitos, sendo a gravidade dos métodos evidenciada, a partir da denúncia e notificação ${ }^{15,20}$. Cabe 
enfatizar que aspectos ligados à transmissão geracional da violência infanto-juvenil podem interferir de forma negativa no processo de formação educacional, desempenho e desenvolvimento psicossocial. Crianças e adolescentes vitimizados aprendem comportamentos agressivos, que tendem a reproduzir ao longo de suas vidas, perpetuando o ciclo da vitimização - agressão ${ }^{21}$. O alto índice de notificação da violência física observada nesta e em outras pesquisas pode ser justificado pela presença de marcas corporais visíveis, facilitando a identificação, em relação às outras formas de violência, que requer investigação mais minuciosa para identificação do problema ${ }^{13}$.

Para a violência psicológica, os achados de Feira de Santana estão em consonância com a literatura, que mostra a alta frequência dessa vitimização, sob as diversificadas formas de ameaças, manifestações verbais, constrangimentos, humilhações, entre outras ${ }^{22}$. Na presente pesquisa, essa violência foi mais prevalente no sexo feminino e nas faixas da infância, cujos agressores foram familiares e conhecidos, sugerindo a presença de vitimização intra e extrafamiliar. Apesar da dificuldade para a elucidação da violência psicológica, visto que não expressa evidência imediata, o agravo psicológico pode estar direta ou indiretamente envolvido na gênese daquele que suscitou o atendimento. Este tipo de violência compromete a autoimagem e estima, provocando sentimentos de desamparo e minusvalia ${ }^{23}$. Durante as consultas, os profissionais podem ser testemunhas de ameaças e atitudes de depreciação direcionadas às vítimas, por familiares ou conhecidos, indicando a necessidade de atenção ao caso e investigação da situação.

No quesito violência sexual, a literatura mundial aponta como um fenômeno feminino, sendo as meninas as principais vítimas do abuso e exploração, independente da faixa etária. Entretanto, vale considerar que pesquisas realizadas em diversos contextos e países, com dados de Instâncias de Referência para denúncias mostram consideráveis proporções de acometimento do sexo masculino, nas faixas da infância ${ }^{24,25}$. No presente estudo, a vitimização foi predominantemente feminina, com altas proporções nas faixas da infância e da adolescência, com elevada ocorrência de manifestação grave (estupro, com penetração genital), com muitas vítimas direcionadas ao atendimento na Rede de Serviços Especializados, para procedimentos, exames e profilaxias (HIV, DST e Hepatite B), além dos encaminhamentos às Instâncias do Sistema de Garantia de Direitos (Conselhos Tutelares, Delegacias especializadas).
Pesquisas revelam que a vitimização sexual de crianças e adolescentes costuma estar ligada a questões de gênero, marcada pelo autoritarismo, moldada pelas condições de vida das famílias, suas relações afetivas e da sexualidade e pautada em aspectos culturais e da representação social do masculino e feminino. As motivações dos agressores não têm origem apenas no desejo, mas abrange questões relacionadas ao poder do indivíduo mais experiente, que assume a posição de dominador, em relação ao mais jovem e inexperiente ${ }^{26,27}$. Crianças e adolescentes encontram-se em fase de desenvolvimento psicossocial, cuja imaturidade não permite compreender a teia criada pelo agressor (familiar ou pessoa de confiança, adulto ou adolescente de maior idade), o qual impõe autoridade, para concretizar a sedução e a prática sexual.

Em Feira de Santana, os resultados apontaram alta frequência de agressor sexual familiar e conhecido, achados que mostram o impacto do ambiente social mais amplo, além dos limites da família, sugerindo a necessidade de sensibilização e envolvimento da família e da comunidade, nas ações e programas de prevenção e proteção eficientes, visto a alta vulnerabilidade desse grupo populacional.

No que concerne à negligência, os dados do Sistema VIVA de Feira de Santana mostraram baixa proporção de registro, comparada com outros tipos de violência (física, sexual e psicológica), possivelmente, pela negligência estar associada aos demais tipos de violência, ou mesmo pela dificuldade na identificação das múltiplas situações que estão classificadas como negligência. De acordo com a literatura, este tipo de agravo exige alguns elementos fundamentais para identificação, como o conhecimento dos fatores determinantes, especialmente em contextos de pobreza, isolamento e privação social, onde o sistema familiar apresenta outras carências e problemas, relacionados à história de vida dos pais ${ }^{28}$. $\mathrm{Na}$ perspectiva ecossistêmica, o fenômeno da negligência reflete ausência ou insuficiência dos cuidados básicos da família dedicados às crianças e adolescentes ${ }^{29}$. Do ponto de vista da saúde pública, ressalta-se a interação entre atos violentos, negligentes ou omissos e respectivas consequências que, mesmo quando não fatais, geram danos à saúde, podendo causar traumas, sequelas e incapacidades, temporárias ou permanentes; provocando sofrimentos físicos e emocionais, os quais demandam atendimento clínico e psicossocial, gerando aumento dos gastos para o Setor Saúde ${ }^{30,31}$. 
Os resultados mostrados nesta e em outras pesquisas apontam para o impacto da vitimização intra e extrafamiliar, com a participação dos diferentes membros da família, assim como pessoas de confiança, do ciclo de convivência da vítima e família, enfatizando a magnitude do fenômeno e, ao mesmo tempo, dificuldades operacionais, para investigação, diagnóstico e atuação preventiva e de controle. Vale ressaltar que, na medida em que a unidade familiar e o cortejo de amigos, vizinhos, pessoas de confiança estão envolvidos, sendo a vítima uma criança ou adolescente, o processo de revelação dos acontecimentos pode delongar muito tempo, contribuindo para graves consequências e perpetuação do ciclo de vitimização e revitimização ${ }^{10}$.

Segundo registros do Sistema VIVA de Feira de Santana, o domicílio se destacou para a ocorrência de eventos violentos, nas faixas da infância ( $\leq 11$ anos), da mesma forma que o meio social, de convívio das vítimas, ratificando resultados semelhantes verificados em outros contex$\operatorname{tos}^{31,32}$. As peculiaridades das famílias, tais como composição, condições e estilo de vida, assim como do meio ambiente, sofrem o impacto das mudanças sociais, culturais e econômicas, redefinindo a dinâmica dos relacionamentos e agrupamentos $^{33}$.

$\mathrm{Na}$ discussão dos agressores responsáveis pelos diferentes tipos de violência, faz-se pertinente abordar questões de gênero, tendo em vista a relação entre o comportamento de meninos e meninas e a influência no estilo de vida, onde são estabelecidos estereótipos, com atitudes diferenciadas entre os sexos. A maior ocorrência de agressões físicas entre meninos pode estar relacionada aos aspectos simbólicos da masculinidade, onde crianças e adolescentes reproduzem atitudes dos adultos, modelos de identificação, baseados em valores e papéis de gênero ${ }^{34}$.

No presente estudo, as proporções de vitimização foram semelhantes entre os sexos, no geral das ocorrências, entretanto, divergiram segundo o tipo de violência. Os meninos foram mais acometidos pela violência física, enquanto as meninas, pela violência psicológica e sexual, sendo que a violência física ocorreu, principalmente, em adolescentes e as violências sexual e psicológica em crianças. Esses resultados concordam com pesquisas realizadas com registros de Instâncias de Garantia de Direitos em diferentes contextos, que observaram resultados semelhantes ${ }^{35,36}$.

Entre os determinantes da vitimização de crianças e adolescentes, cabe salientar a importância dos adultos de referência, quanto às pró- prias condutas, considerando a missão de garantir proteção e formação familiar, educação formal (escola) e convívio social, pautados em princípios universais de direitos humanos e deveres, no gozo da cidadania plena ${ }^{12,37}$. Ainda nos aspectos voltados ao compromisso dos adultos, vale assinalar a importância do controle social, com ressalva ao importante papel da mídia (televisão e internet), na disseminação de informações direcionadas à infância e adolescência, com massiva disseminação de mensagens que estimulam atitudes agressivas (jogos, filmes e outras atividades), reforçando a presença das múltiplas formas de violência, como meios de comunicação e intermediação de conflitos entre os indivíduos e o ambiente social ${ }^{13,16}$.

O tipo de atendimento dispensado pelo setor saúde às vítimas de violência é reflexo da gravidade das lesões. Os resultados do presente estudo permitiram apontar que, dentre os casos em que foi efetuado algum encaminhamento no setor saúde, a maior parte tenha recebido atendimento em nível ambulatorial e em 35\% a internação tenha sido solicitada e 15\% tenham evoluído para óbito. Estes dados mostram a gravidade do problema e a necessidade de medidas de prevenção e proteção e apontam para a importância do Sistema de Vigilância Epidemiológica, levantando indicadores, mostrando a magnitude e a tendência dos agravos e subsidiando o planejamento e a aplicação de estratégias apropriadas e coerentes com a realidade social ${ }^{12,15}$.

No quesito encaminhamentos, estudos assinalam a importância da integração entre múltiplos setores, configurando o trabalho em Rede, baseado na cooperação mútua que, por meio da articulação político-institucional pode negociar e partilhar recursos e responsabilidades, de acordo com demandas e missão organizacional. Essa estratégia possibilita troca de informações e formulação de políticas direcionadas à implementação de práticas e projetos comuns, que contribuem para a integralidade da atenção, defesa e garantia de direitos a crianças, adolescentes e famílias em situação de vulnerabilidade ${ }^{37}$. A interlocução da Rede, durante o processo de notificação, permite estabelecer um circuito fundamental, na proteção das vítimas e orientação das famílias, uma vez que essas instituições têm o poder de determinar, por força de lei, as ações de atendimento e de responsabilização. Feira de Santana corrobora com o preconizado na rede de proteção às crianças e adolescentes: os casos de violências atendidos na Rede de Saúde e notificação no Sistema VIVA, foram encaminhados aos 
Conselhos Tutelares (Instituição de referência do Sistema de Garantia de Direitos), revelando esclarecimento sobre o processo de notificação e credibilidades nessas instâncias.

Como limitações, relata-se o fato do VIVA não captar a parcela de atendimento nos serviços privados do município (que pouco notificam), a insuficiência de quesitos relacionados às informações dos agressores e a subnotificação por parte dos profissionais de saúde.

\section{Considerações finais}

O Sistema de Informação de Violências e Acidentes/VIVA contribui para ampliar o conheci- mento da violência-infanto-juvenil, na perspectiva do Setor Saúde, auxiliando no levantamento de indicadores, assim como subsidiando a ampliação das políticas, programas e práticas voltadas à prevenção, enfrentamento da violência e redução de danos.

A Rede de atenção integral às vítimas de violência vem se consolidando e garantindo o acesso aos serviços, cabendo enfatizar a importância da articulação do Sistema de Saúde com a Rede de Atendimento e Defesa dos Direitos, diante do desafio de identificar, notificar e encaminhar os casos, ampliando a resolutividade e garantindo proteção e defesa de direitos, especialmente nos grupos mais vulneráveis.

\section{Colaboradores}

CS Souza, MCO Costa, SG Assis, JO Musse, CN Sobrinho e MTR Amaral participaram igualmente de todas as etapas de elaboração do projeto.

\section{Agradecimentos}

À FAPESB pelo financiamento do projeto. 


\section{Referências}

1. Organização Mundial de Saúde (OMS). Relatório Mundial sobre Violência e Saúde. Genebra: OMS 2002.

2. Brasil. Ministério da Saúde (MS). Secretaria de Vigilância em Saúde. Impacto da violência na saúde dos Brasileiros. Ministério da Saúde, Secretaria de Vigilância em Saúde. Brasília: MS; 2005.

3. Schraiber LB, D'oliveira AFPL, Couto MT. Violência e saúde: estudos científicos recentes. Rev Saude Publica 2006, 40(N. Esp):112-120.

4. Minayo MCS. A inclusão da violência na agenda da saúde: trajetória histórica. Cien Saude Colet 2007; 11(Supl.):1259-1267.

5. Malta DC, Mascarenhas MDM, Bernal RTI, Andrade SSCA, Neves ACM, Melo EM, Silva Junior JB. Causas externas em adolescentes: atendimentos em serviços sentinelas de urgência e emergência nas Capitais Brasileiras - 2009. Cien Saude Colet 2012; 17(9):2291-2304.

6. Bezerra SC. Estatuto da Criança e do Adolescente: marco da proteção integral. In: Brasil. Ministério da Saúde. Violência faz mal à saúde. Brasília: MS; 2004. p. 17-22.

7. Brasil. Lei 8.069 , de 13 de julho de 1990. Dispõe sobre o Estatuto da Criança e do Adolescente e dá outras providências. Diário Oficial da União 1990; 16 jul.

8. Belon AP, Silveira NYJ, Barros MBA, Baldo C, Silva MMA. Atendimentos de emergência a vítimas de violências e acidentes: diferenças no perfil epidemiológico entre o setor público e o privado. VIVA - Campinas/SP, 2009. Cien Saude Colet 2012; 17(9): 2279-2290.

9. Brasil. Ministério da Saúde (MS). Secretaria de Vigilância em Saúde. Departamento de Análise de Situação de Saúde. Viva: vigilância de violências e acidentes, 2008 e 2009. Brasília: MS; 2010.

10. Gonçalves HS, Ferreira AL. A notificação da violência intrafamiliar contra crianças e adolescentes por profissionais de saúde. Cad Saude Publica 2002; 18(1):315-319.

11. Brasil. Ministério da Saúde (MS). Secretaria de Assistência à Saúde. Notificação de maus-tatos contra crianças e adolescentes pelos profissionais de saúde: um passo a mais na cidadania em saúde. Brasília: MS; 2002.

12. Ricas J, Donoso MTV, Gresta MLM. A violência na infância como uma questão cultural. Texto Contexto Enferm 2006; 15(1):151-154.

13. Ferreira AL, Souza ER. Análise de indicadores de avaliação do atendimento a crianças e adolescentes em situação de violência. Cad Saude Publica 2008; 24(1):28-38.

14. Brasil. Ministério da Saúde (MS). Conselho Nacional de Saúde. Resolução no 196 de 10 de outubro de 1996. Diretrizes e Normas Regulamentadoras de Pesquisas Envolvendo Seres Humanos. Diário Oficial da União 1996; 16 out.

15. Assis SG, Avanci JQ, Pesce RP, Pires TO, Gomes DL. Notificações de violência doméstica, sexual e outras violências contra crianças no Brasil. Cien Saude Colet 2012; 17(9):2305-2317.
16. Minayo MCS. Violência contra crianças e adolescentes: questão social, questão de saúde. Rev. Bras. Saúde Mater Infantil 2001; 1(2):91-102.

17. Deslandes SF, Lemos MP. Construção participativa de descritores para avaliação dos núcleos de prevenção de acidentes e violência, Brasil. Rev Panam Salud Publica 2008; 24(6):441-448.

18. Minayo MCS. Violência e Saúde. Rio de Janeiro: Fiocruz; 2006.

19. Malta DC, Mascarenhas MDM, Bernal RTI, Viegas APB, Sá NNB, Silva Junior JB. Acidentes e violência na infância: evidências do inquérito sobre atendimentos de emergência por causas externas - Brasil, 2009. Cien Saude Colet 2012; 17(9):2247-2258.

20. Junqueira MF. Abuso sexual da criança: Contextualização. Rev Pediatr Moderna 1998; 24:432-436.

21. Kanul F, Ramírez MA. Family violence and child abuse in Latin America and the Caribbean: the cases of Colombia and Mexico. Washington: Inter-American Development Bank; 2005.

22. Krug EG, Dahlberg LL, Mercy J A, Zwi AB, Lozano $\mathrm{R}$, editors. World report on violence and health. Geneva: World Health Organization (WHO); 2002.

23. Assis SG, Avanci JQ. Abuso psicológico e desenvolvimento infantil. In: Brasil. Mistério da Saúde (MS). Violência faz mal à saúde. Brasília: MS; 2004.

24. Lavergne C, Tourigny M. Incidence de l'abusetla negligence em versles enfants: recension desécrits. Criminologie 2000; 33(1):47-72.

25. Trocmé NM, Tourigny M, Maclaurin B, Fallon B. Major findings from the Canadian incidence study of reported child abuse and neglect. Child Abuse Negl 2003; 27(12):1427-1439.

26. Ribeiro MA, Ferriani MGC, Reis JN. Violência sexual contra crianças e adolescentes: características relativas à vitimizaçãonas relações familiares. Cad Saude Publica 2004; 20(2):456-464.

27. Faleiros VP, Faleiros ETS. Circuito e curtos - circuitos: atendimento, defesa e responsabilização do abuso sexual contra crianças e adolescentes. São Paulo: Veras; 2001.

28. Morais EP, Eidt OR. Conhecendo para evitar: a negligência nos cuidados de saúde com crianças e adolescentes. Rev Gaúcha Enferm 1999; 20:6-21.

29. Barudy J. La douleur invisible de l'enfant: approche éco-systémique de la maltraitance. Ramonville SaintAgne: Éres; 1997.

30. Costa MCO, Bigras M. Mecanismos pessoais e coletivos de proteção e promoção da qualidade de vida para a infância e adolescência. Cien Saude Colet 2007; 12(5):1101-1109.

31. Mello-Jorge MHP. Violência como problema de saúde pública. Ciência e Cultura 2002; 54(1):52-53. De Antoni C, Koller SH. Vulnerabilidade e resiliência familiar. Um estudo com adolescentes que sofreram maus tratos intrafamiliares. Estud. psicol. (Natal) 2000; 5(2):347-381.

32. Habigzang LF, Caminha RM. Abuso sexual contra crianças e adolescentes: Conceituação e intervenção clínica. São Paulo: Casa do Psicólogo; 2004. 
33. Salvagni EP, Wagner MB. Estudos de caso-controle para desenvolver e estimar a validade discriminate de um questionário de avaliação de abuso sexual em crianças. J Pediatr 2006; 82(6):431-436.

34. Souza ER. Masculinidade e violência no Brasil: contribuições para a reflexão no campo da saúde. Cien Saude Colet 2005; 10(1):59-70.

35. Costa MCO, Carvalho RC, Bárbara JFR, Santos CAST, Gomes WA, Sousa HL. O perfil da violência contra crianças e adolescentes, segundo registros de Conselhos Tutelares: vítimas, agressores e manifestações de violência. Cien Saude Colet 2007; 12(5):1129-1141.

36. Johnson G, Kent G, Leather J. Strengthening the parent-child relationship: a review of family interventions and their use in medical settings. Child: Care, Health Dev 2005; 31(1):25-32.

Artigo apresentado em 01/09/2013

Aprovado em 23/09/2013

Versão final apresentada em 30/09/2013 